\title{
Pore scale investigation of gaseous mixture flow in porous anode of solid oxide fuel cell
}

Zheng Dang, Han $\mathrm{Xu}^{*}$

Dept. of Building Environment and Energy Engineering, Xi'an Jiaotong University,

Xi'an 710049, China

* Corresponding Author, E-mail: xuhanxh@mail.xjtu.edu.cn

(C) 2016. This manuscript version is made available under the Elsevier user license http://www.elsevier.com/open-access/userlicense/1.0/ 


\section{Abstract:}

A two-fluid finite-difference Lattice Boltzmann (LB) model is developed for multi-component flow. Then, a pore scale LB model is further developed for multi-component flow in porous media with heterogeneous microstructure. Thus, the present LB model is independent on any structure statistical parameters of the porous media, which is contrary to the numerical methods assuming the porous media is homogenous. Compared with existing LB models for multi-component flow in the solid oxide fuel cell (SOFC) anode, the present LB model is capable of simulating mixture flow with larger ratios of molecular weights, thus it is qualified to meet the fuel flexibility of the SOFC. By using this model, the mass transfer of gaseous mixture flow in a SOFC anode is studied and the local molar fraction distributions of gaseous mixture are obtained within the irregular porous anode directly. The influences of anode microstructure, dimensionless reaction flux and fuel composition on anode performance are investigated. The quantitative relationship between the porous microstructure and anode performance is obtained.

Keywords: Solid oxide fuel cell; Porous anode; Lattice Boltzmann method; Pore scale; Microstructure; Mass transfer 


\section{Introduction}

Solid oxide fuel cell (SOFC) has been considered as one of the most promising power generation devices[1] due to its high electrical efficiency[2] and fuel flexibility[3]. However, the design and manufacture of durable electrodes with high performance is a technological barrier to the commercialization of SOFC[4]. It is crucial to study various transport processes and reactions in electrodes to improve their performance and durability[5]. Notably, the momentum and heat transport in porous electrodes, electronic and ionic transport in electronic and ionic conductors and reaction characteristic at chemical/electrochemical active sites are all significantly affected by the multi-component mass transport existing in pore phase, since it determines the concentration distribution of each species in porous electrodes. Therefore, the multi-component mass transport within porous electrodes plays a key

role in cell performance, especially at high current densities[6], and a detailed understanding of multi-component mass transport is the fundamental of establishing a comprehensive mathematical model of SOFC.

Concentration overpotential is often employed to describe mass transport-related losses of SOFC electrodes. The concentration overpotential of cathode is negligible compared to that of anode[7]. Moreover, when hydrocarbon fuel is adopted, the transport processes and reactions in the anode are much more complicated and important than that in the cathode[8,9]. Consequently, plenty of works have been devoted to the performance improvement of SOFC anodes[10,11].

SOFC anodes are usually porous microstructures with sub-micron-sized pores, 
the heterogeneity of which significantly affects the performance and durability of anodes. It is important to find the quantitative relationship between the anode microstructure and its performance[12]. Due to the limitation of expensive equipment and measuring techniques, it is difficult to obtain distributions of related physical quantities in the heterogeneous porous anodes by experiments under high temperature conditions. In contrast, numerical simulation is a competent alternative. The extended Fick's model (extended FM), Stefan-Maxwell model (SMM) and dusty-gas model (DGM) are extensively employed to simulate the mass transport in previous studies of SOFC[13-16]. However, they are not qualified to simulate the gas transport in the porous anode at micro/meso-scale. Moreover, in these models, the heterogeneity of the electrode microstructure is neglected and hence structure statistical parameters and flow empirical relations have to be employed, which extremely limits their applications.

To fully understand the mass transport in porous anodes, modeling methods considering the micro/meso-scale effect and the microstructure heterogeneity are required, which are usually named as pore scale modeling methods. Lattice Boltzmann (LB) method is thought to be a qualified candidate, since it is deduced from kinetic theory and hence is capable of solving transport processes at micro/meso-scale. Besides, it is very competitive for modeling the mass transport in complex geometries, such as porous structures, because of the simple bounce-back rule for no-slip boundary condition. Several studies have adopted various pore scale LB models to investigate the mass transport in SOFC anodes[17-21]. However, all 
these models suffer the drawbacks of the existing multi-component LB models. Some of them have adopted single-fluid LB model employing single relaxation time which is restricted to unity Prandtl and Schmidt numbers for the multi-species flow $[17,18]$. Others have adopted a relatively more accurate two-fluid LB model[19-21], in which viscosity and diffusion coefficients can be varied independently by changing the cross- and self-collision relaxation times and hence variable Prandtl and Schmidt numbers can be considered. However, the maximum ratio of molecular weights that can be accurately simulated by this two-fluid LB model is limited to be below 9[22], which undoubtedly limits the applicability of this model, and cannot simulate the transport processes in SOFCs fed by other common fuels

In our previous study[6,23], a coupled LB model was developed to simulate the mass transport in porous anode, which employed the LB model proposed by McCracken and Abraham[22] for multi-component flow and a representative elementary volume (REV) scale LB algorithm based on the Brinkman equation for flows in porous media, and this model is proved to be more accurate than extended FM, SMM and DGM. In present study, a new multi-component LB model extending the applicability of model developed by McCracken and Abraham[22] to mixture flow with ratio of molecular weights above 9 is established, which is not only based on two-fluid theory, but also capable of meeting the fuel flexibility of the SOFC. Moreover, a pore scale LB model is further developed based on the heterogeneous microstructure of porous anode to investigate the multi-component mass transport in a SOFC anode, so as to investigate the quantitative relationship between the porous 
microstructure and anode performance. Furthermore, the influences of anode microstructure, dimensionless reaction flux and fuel composition on anode performance are discussed, in terms of local concentration distributions, molar fraction of reactive gas along the primary mass transport direction, and concentration overpotential.

\section{Lattice Boltzmann model}

\subsection{General description}

The transport processes and electrochemical reactions in the SOFC anode are illustrated thoroughly in Ref.[23]. As shown in Fig. 1(a), hydrogen from the fuel channel diffuses to the three phase boundary (TPB), i.e., the interface among electronic conductor, ionic conductor and gas species, then reacts with the oxygen ion diffusing from the electrolyte to generate steam and electrons (Eq. (1)). Finally, the electrons formed in the hydrogen oxidation reaction are collected by the current collector and migrate to the cathode via external circuit to meet the electrical load.

$$
\mathrm{H}_{2(\mathrm{TPB})}+\mathrm{O}_{(\mathrm{TPB})}^{2-} \rightarrow \mathrm{H}_{2} \mathrm{O}_{(\mathrm{TPB})}+2 \mathrm{e}^{-}
$$

The anode is usually a porous cermet composed of electronic conductor and ionic conductor. A scanning electron microscope (SEM) image of the microstructure of an SOFC anode made up of Ni and scandium stabilized zirconia (SSZ) is presented in Fig. 1(b). By binarization processing, a binary image of the anode microstructure is then obtained (Fig. 1(c)). Higher contrast between the solid and pore phases is obtained in Fig. 1(c) compared with Fig. 1(b), which is used as the geometric input 
condition of the pore scale simulation. The imaging and description of the porous anode microstructure will be introduced in Sec. 2.3 in detail.

Since the present study is mainly focused on the multi-component mass transport in the anode, the anode/electrolyte interface is assumed to be the TPB for simplicity, which is a commonly employed assumption in relevant literatures[24-26]. The relevant physical model and boundary conditions are presented in Fig. 2. At the fuel channel/anode interface (i.e., $x=0$ ), the mixture total concentration and molar fraction of each species are specified by Eq. (2),

$c_{\mathrm{m}, \mathrm{bulk}}=c_{\mathrm{T}} \cdot y_{\mathrm{m}, \mathrm{bulk}}$

where $\mathrm{m}$ represents the $\mathrm{m}^{\text {th }}$ species, $c$ is the molar concentration, $c_{\mathrm{T}}$ is the total molar concentration of mixture and $y$ is the molar fraction. At the anode/electrolyte interface (i.e., $x=\mathrm{L}$ ), the molar flux of each species is specified by Eq. (3) based on the current drawn by the SOFC, $J_{\mathrm{H}_{2}}=\frac{i}{2 F}, J_{\mathrm{H}_{2} \mathrm{O}}=-J_{\mathrm{H}_{2}}$

where $J$ and $i$ are molar flux and average current density at TPB, respectively. Since the thickness of the anode is far less than the length of the cell and this paper primarily focuses on the mass transport along the thickness of electrodes ( $x$ direction), periodic boundaries in Eq. (4) are employed at both the top and the bottom of the computational domain and a reasonable height $H$ is adopted to provide a realistic representation of the porous anode,

$\left.\psi_{\mathrm{m}}\right|_{\mathrm{z}=0}=\left.\psi_{\mathrm{m}}\right|_{\mathrm{z}=\mathrm{H}}$

where $\psi$ represents the relevant physical quantity. The solid obstacles are 
impermeable and no-slip boundary conditions are applied at the obstacle surfaces,

$$
\frac{\partial c_{\mathrm{m}}}{\partial \boldsymbol{n}}=0
$$

where $\boldsymbol{n}$ is the unit normal perpendicular to obstacle surfaces.

The concentration overpotential of the anode is described as

$$
\eta_{\text {con }}=-\frac{R T}{2 F} \ln \left(\frac{y_{\mathrm{H}_{2}, \mathrm{~A} / \mathrm{E}} y_{\mathrm{H}_{2} \mathrm{O}, \mathrm{bul} k}}{y_{\mathrm{H}_{2}, \mathrm{bulk}} y_{\mathrm{H}_{2} \mathrm{O}, \mathrm{A} / \mathrm{E}}}\right)
$$

\subsection{LB model for multi-component flow}

LB methods describe the fluid flow at each lattice point of a numerical grid by two steps: streaming and collision. In the streaming step, particles move along specified lattice directions; in the collision step, particles of the same or of different species interact with each other as they arrive at the lattice point from neighboring points. The Boltzmann equation for a binary system based on the two-fluid theory is as follows (taking the $\sigma^{\text {th }}$ species as an example)

$$
\frac{\partial f^{\sigma}}{\partial t}+\boldsymbol{\xi} \cdot \frac{\partial f^{\sigma}}{\partial \boldsymbol{r}}+\boldsymbol{a}^{\sigma} \cdot \frac{\partial f^{\sigma}}{\partial \boldsymbol{\xi}}=J^{\sigma \sigma}+J^{\sigma s}
$$

where $\sigma$ and $s$ are two different species, $\boldsymbol{\xi}$ is the velocity, and $\boldsymbol{a}$ is the acceleration. In the two-fluid theory, the collision is separated into the self-collision and the cross-collision. The self-collision term $J^{\sigma \sigma}$ represents the effect of collisions between particles of the same species. The cross-collision term $J^{\sigma s}$ represents the effect of collisions between particles of different species. The cross- and self-collision terms are calculated independently, thus the viscosity and diffusion coefficients can be varied independently by changing the cross- and self-collision relaxation times. It is contrary to most existing single-fluid LB models restricted to unity Prandtl and 
Schmidt numbers.

In our previous study[6,23], the multi-component LB model based on the two-fluid model initially proposed by Luo and Girimaji[27,28] and extended to fluids with different molecular weights by using the interpolation scheme to solve the streaming process proposed by McCracken and Abraham[22] is employed. However, the interpolation is cumbersome, and it is proved that the ratio of molecular weights should be below 9 in order to accurately capture the physics of binary diffusion owing to the numerical diffusion. Viewed from another perspective, Eq. (7) is a partial differential equation, which can be discretized by using finite-difference method (FDM)[29], finite-volume method (FVM)[30] and finite-element method (FEM)[31] . In this paper, a two-fluid finite-difference LB model for multi-component flow is developed, which employs the two-fluid theory and FDM to describe the collision and the streaming, respectively. Therefore, this model is capable of simulating the multi-component flow with arbitrary Pr and Sc numbers. Besides, it is much more convenient to implement and has a better numerical stability compared to the interpolation scheme proposed by McCracken and Abraham[22].

Because different species have different streaming distance in the same time step $\delta_{t}$, the lattice velocity of each species is (assuming that the molecular weight of the $\sigma^{\text {th }}$ species is the smallest)

$$
c^{\sigma}=\frac{\delta_{x}}{\delta_{t}}, c^{s}=\sqrt{\frac{M^{\sigma}}{M^{s}}} c^{\sigma}
$$

The discrete velocity can be described as 


$$
\boldsymbol{e}_{\alpha}^{\sigma}= \begin{cases}(0,0), & \left(e_{\alpha}^{\sigma}\right)^{2}=0 \\ c^{\sigma}(\cos [(\alpha-1) \pi / 2], \sin [(\alpha-1) \pi / 2]), & \left(e_{\alpha}^{\sigma}\right)^{2}=\left(c^{\sigma}\right)^{2} \\ \sqrt{2} c^{\sigma}(\cos [(2 \alpha-1) \pi / 4], \sin [(2 \alpha-1) \pi / 4]), & \left(e_{\alpha}^{\sigma}\right)^{2}=2\left(c^{\sigma}\right)^{2}\end{cases}
$$

The speed of sound of each species is

$$
c_{s}^{\sigma}=\frac{c^{\sigma}}{\sqrt{3}}, c_{s}^{s}=\frac{1}{\sqrt{3}} \sqrt{\frac{M^{\sigma}}{M^{s}}} c^{\sigma}
$$

By discretizing Eq. (7), the following equation is obtained:

$$
\frac{\partial f_{\alpha}^{\sigma}}{\partial t}+\boldsymbol{e}_{\alpha}^{\sigma} \cdot \nabla f_{\alpha}^{\sigma}=J_{\alpha}^{\sigma \sigma}+J_{\alpha}^{\sigma s}+F_{\alpha}^{\sigma}
$$

where

$$
\begin{aligned}
& J_{\alpha}^{\sigma \sigma}=-\frac{1}{\tau^{\sigma}}\left[f_{\alpha}^{\sigma}-f_{\alpha}^{\sigma(0)}\right] \\
& f_{\alpha}^{\sigma(0)}=\left[1+\frac{1}{R_{\sigma} T}\left(\boldsymbol{e}_{\alpha}^{\sigma}-\boldsymbol{u}\right) \cdot\left(\boldsymbol{u}^{\sigma}-\boldsymbol{u}\right)\right] f_{\alpha}^{\sigma(e q)} \\
& f_{\alpha}^{\sigma(e q)}=\rho^{\sigma} \omega_{\alpha}\left[1+\frac{\boldsymbol{e}_{\alpha}^{\sigma} \cdot \boldsymbol{u}}{R_{\sigma} T}+\frac{\left(\boldsymbol{e}_{\alpha}^{\sigma} \cdot \boldsymbol{u}\right)^{2}}{2\left(R_{\sigma} T\right)^{2}}-\frac{u^{2}}{2 R_{\sigma} T}\right] \\
& J_{\alpha}^{\sigma s}=-\frac{1}{\tau_{D}} \frac{\rho^{s}}{\rho} \frac{f_{\alpha}^{\sigma(e q)}}{R_{\sigma} T}\left(\boldsymbol{e}_{\alpha}^{\sigma}-\boldsymbol{u}\right) \cdot\left(\boldsymbol{u}^{\sigma}-\boldsymbol{u}^{s}\right) \\
& F_{\alpha}^{\sigma}=\rho^{\sigma} \omega_{\alpha} \frac{\boldsymbol{e}_{\alpha}^{\sigma} \cdot \boldsymbol{a}^{\sigma}}{R_{\sigma} T}
\end{aligned}
$$

$\tau^{\sigma}$ is the self-collision relaxation time controlling the kinematic viscosity, and $\tau^{\sigma s}$ is the cross-collision relaxation time determining the inter-species diffusivities.

The density and velocity of each species are moments of distribution functions:

$$
\begin{aligned}
& \rho^{\sigma}=\sum_{\alpha} f_{\alpha}^{\sigma}=\sum_{\alpha} f_{\alpha}^{\sigma(0)} \\
& \rho^{\sigma} \boldsymbol{u}^{\sigma}=\sum_{\alpha} f_{\alpha}^{\sigma} \boldsymbol{e}_{\alpha}^{\sigma}=\sum_{\alpha} f_{\alpha}^{\sigma(0)} \boldsymbol{e}_{\alpha}^{\sigma}
\end{aligned}
$$

The density and velocity of the mixture are defined as 
$\rho=\rho^{\sigma}+\rho^{s}$

$\rho \boldsymbol{u}=\rho^{\sigma} \boldsymbol{u}^{\sigma}+\rho^{s} \boldsymbol{u}^{s}$

The pressure of each species and the mixture are

$$
\begin{aligned}
& p^{\sigma}=\rho^{\sigma}\left(c_{s}^{\sigma}\right)^{2} \\
& p=p^{\sigma}+p^{s}=\frac{\left(c^{\sigma}\right)^{2}}{3}\left(\rho^{\sigma}+\rho^{s} \frac{M^{\sigma}}{M^{s}}\right)=\frac{1}{3}\left(\rho^{\sigma}+\rho^{s} \frac{M^{\sigma}}{M^{s}}\right)\left(\frac{\delta_{x}}{\delta_{t}}\right)^{2}
\end{aligned}
$$

Eq. (7) can be divided into two parts

Collision: $\frac{\partial f_{\alpha}^{\sigma}}{\partial t}=J_{\alpha}^{\sigma \sigma}+J_{\alpha}^{\sigma s}+F_{\alpha}^{\sigma}$

Streaming: $\frac{\partial f_{\alpha}^{\sigma}}{\partial t}+\boldsymbol{e}_{\alpha}^{\sigma} \cdot \nabla f_{\alpha}^{\sigma}=0$

Eq. (23) is discretized by an explicit first-order Euler scheme in time space

$f_{\alpha}^{\sigma+}=f_{\alpha}^{\sigma}+\delta_{t} J_{\alpha}^{\sigma \sigma}+\delta_{t} J_{\alpha}^{\sigma s}+\delta_{t} F_{\alpha}^{\sigma}$

Eq. (24) is discretized by a second-order Lax-Wendroff scheme in time space on one set of grid as shown in Eq. (26), which is much easier to implement than the interpolation scheme needing $N$ sets of grid for $N$ species[22,32].

$$
\begin{aligned}
f_{\alpha}^{\sigma}\left(\boldsymbol{x}, t+\delta_{t}\right) & =f_{\alpha}^{\sigma+}(\boldsymbol{x}, t)-\delta_{t}\left(\boldsymbol{e}_{\alpha}^{\sigma} \cdot \nabla\right) f_{\alpha}^{\sigma+}(\boldsymbol{x}, t) \\
& +\frac{1}{2} \delta_{t}^{2}\left(\boldsymbol{e}_{\alpha}^{\sigma} \cdot \nabla\right)^{2} f_{\alpha}^{\sigma+}(\boldsymbol{x}, t)+o\left(\delta_{t}^{3}\right)
\end{aligned}
$$

Substituting $f_{\alpha}{ }^{\sigma+}$ given by Eq. (25) into Eq. (26), and expanding the left-hand side in Taylor series in $\delta_{t}$ up to $O\left(\delta_{t}^{2}\right)$, the equation can be rewritten as

$$
D_{\alpha}^{\sigma} f_{\alpha}^{\sigma}=J_{\alpha}^{\sigma \sigma}+J_{\alpha}^{\sigma s}+F_{\alpha}^{\sigma}-\frac{\delta_{t}}{2} D_{\alpha}^{\sigma 2} f_{\alpha}^{\sigma}+o\left(\delta_{t}^{2}\right)
$$

where $D_{\alpha}=\partial_{t}+\left(\boldsymbol{e}_{\alpha} \cdot \nabla\right)$.

By means of Chapman-Enskog analysis, Eq. (27) can be rewritten in consecutive orders of the parameter $\kappa$ as 


$$
\begin{aligned}
& \kappa^{0}: f_{\alpha}^{\sigma(0)}=\left[1+\frac{1}{R_{\sigma} T}\left(\vec{e}_{\alpha}^{\sigma}-\vec{u}\right) \cdot\left(\vec{u}^{\sigma}-\vec{u}\right)\right] f_{\alpha}^{\sigma(e q)} \\
& \kappa^{1}: \delta_{t} D_{\alpha}^{\sigma(0)} f_{\alpha}^{\sigma(0)}=-\frac{1}{\tau^{\sigma}} f_{\alpha}^{\sigma(1)}+J_{\alpha}^{\sigma s}+\delta_{t} F_{\alpha}^{\sigma} \\
& \kappa^{2}: \delta_{t} \partial_{t 1} f_{\alpha}^{\sigma(0)}+\delta_{t}\left(1-\frac{1}{2 \tau^{\sigma}}\right) D_{\alpha}^{\sigma(0)} f_{\alpha}^{\sigma(1)}+\frac{\delta_{t}}{2} D_{\alpha}^{\sigma(0)} J_{\alpha}^{\sigma s}-\frac{\delta_{t}^{2}}{2} D_{\alpha}^{\sigma(0)} F_{\alpha}^{\sigma} \\
& \quad=-\frac{1}{\tau^{\sigma}} f_{\alpha}^{\sigma(2)}
\end{aligned}
$$

where $\kappa$ is an expansion parameter used in the Chapman-Enskog analysis. Then, the relevant hydrodynamic equations are derived from Eqs. (28), (29) and (30).

Taking zeroth-order moments of Eqs. (29) and (30), the mass conservation equation for each species is deduced as

$$
\partial_{t} \rho^{\sigma}+\nabla \cdot\left(\rho^{\sigma} \boldsymbol{u}^{\sigma}\right)=\frac{1}{2} \nabla \cdot\left[\frac{1}{\tau_{D}} \frac{\rho^{s} \rho^{\sigma}}{\rho}\left(\boldsymbol{u}^{\sigma}-\boldsymbol{u}^{s}\right)\right]
$$

Then, the mass conservation equation for the mixture is obtained:

$$
\partial_{t} \rho+\nabla \cdot(\rho \boldsymbol{u})=0
$$

Taking first-order moments of Eqs. (29) and (30), the momentum conservation equation for each species is obtained as

$$
\begin{aligned}
\partial_{t}\left(\rho^{\sigma} \boldsymbol{u}^{\sigma}\right)+\left(\rho^{\sigma} \boldsymbol{u}^{\sigma} \cdot \nabla\right) \boldsymbol{u}= & -\nabla p^{\sigma}+v^{\sigma} \nabla^{2}\left(\rho^{\sigma} \boldsymbol{u}^{\sigma}\right)+\left(\kappa-\frac{\delta_{t}}{2} \kappa^{2} \partial_{t 0}\right)\left(\rho^{\sigma} \boldsymbol{a}^{\sigma}\right) \\
& -\left(\frac{\kappa}{\delta_{t}}+\frac{1}{2} \kappa^{2} \partial_{t 0}\right) \frac{\rho^{s} \rho^{\sigma}}{\tau_{D} \rho}\left(\boldsymbol{u}^{\sigma}-\boldsymbol{u}^{s}\right)
\end{aligned}
$$

where the viscosity of the $\sigma^{\text {th }}$ species is

$$
v^{\sigma}=\left(\tau^{\sigma}-\frac{1}{2}\right) \delta_{t} c_{s}^{\sigma 2}
$$

Similarly, the momentum conservation equation for the mixture can be deduced as 


$$
\begin{aligned}
\partial_{t}(\rho \boldsymbol{u})+(\rho \boldsymbol{u} \cdot \nabla) \boldsymbol{u} & =-\nabla p+v^{\sigma} \nabla^{2}\left(\rho^{\sigma} \boldsymbol{u}^{\sigma}\right)+v^{s} \nabla^{2}\left(\rho^{s} \boldsymbol{u}^{s}\right) \\
& +\left(\kappa-\frac{\delta_{t}}{2} \kappa^{2} \partial_{t 0}\right)\left(\rho^{\sigma} \boldsymbol{a}^{\sigma}+\rho^{s} \boldsymbol{a}^{s}\right)
\end{aligned}
$$

According to Eq. (31), the following equation is deduced:

$$
\partial_{t} \rho^{\sigma}+\nabla \cdot\left(\rho^{\sigma} \boldsymbol{u}\right)=-\left(1-\frac{1}{2 \tau_{D}}\right) \nabla \cdot\left(-\tau_{D} \delta_{t} p \boldsymbol{d}^{\sigma}\right)
$$

where $\boldsymbol{d}^{\sigma}$ is the diffusion force. Moreover, the diffusion flux could be defined as

$$
\boldsymbol{j}^{\sigma}=-\rho D_{\sigma s} \nabla\left(\frac{\rho^{\sigma}}{\rho}\right)=-D_{\sigma s} \frac{n^{2} M^{\sigma} M^{s}}{\rho} \boldsymbol{d}^{\sigma}
$$

Therefore, the following diffusion equation is obtained:

$$
\partial_{t} \rho^{\sigma}+\nabla \cdot\left(\rho^{\sigma} \boldsymbol{u}\right)=-\nabla \cdot \boldsymbol{j}^{\sigma}
$$

Meanwhile, the diffusivity is derived as

$$
D_{\sigma s}=\frac{p \rho}{n^{2} M^{\sigma} M^{s}}\left(\tau_{D}-\frac{1}{2}\right) \delta_{t}
$$

According to Eq. (39), the diffusivity is independent with the viscosity. Therefore, the model developed in this paper is capable of simulating multi-component flow under arbitrary $\operatorname{Pr}$ and Sc numbers. The above model description is focused on the two-component flow, but the present LB model can be easily extended to $N$-component flow $(N>2)$ by adding relevant cross-collision terms.

\subsection{LB model for multi-component flow in porous media}

The pore scale LB model is employed to simulate the multi-component flow in porous media. Firstly, the detailed geometry of the porous media should be characterized to be the geometry input condition of the pore scale LB model. The SEM image of the cross section of an SOFC anode could be easily obtained and an example is shown in Fig. 1(b). The grayscale of each pixel in the SEM image can be 
obtained first. Then, a threshold of grayscale is selected to divide the pore phase and the solid phase. For the region where the greyscale is less than the threshold, the greyscale is set as 0 (black), and for the remaining, the greyscale is set as 255 (white). The binary image (Fig. 1(c)) is obtained according to the SEM image, in which the black region represents the solid phase (i.e., the mixture of electronic and ionic conductors) and the white region represents the pore phase. From the binary image, a two-dimensional array is defined at each pixel as shown in Eq. (40). The above binarization processing is implemented by Matlab.

$\operatorname{IMAGE}(\boldsymbol{x})=\left\{\begin{array}{l}0, x \in \text { solid phase } \\ 1, x \in \text { pore phase } \\ 2, \boldsymbol{x} \in \text { interface of solid phase and pore phase }\end{array}\right.$

The fluid flow (where $\operatorname{IMAGE}(\boldsymbol{x})=0$ ) is described by the two-fluid finite-difference LB model developed in Sec.2.2. The interfaces of the pore phase and solid phase could be classified into flat, convex and concave boundaries as shown in Fig. 3, and they are dealt with the no-slip bounce-back rule[33].

\section{Results and discussion}

In order to improve the computation efficiency, the LB model developed in this paper is implemented in parallel based on OpenMP (Open Multi-Processing) by a self-writing FORTRAN code. The lattice unit is employed in the LB modelling, and two dimensionless parameters governing the multi-component mass transport in the porous media are porosity $\varepsilon$ and dimensionless reaction flux $J^{*}$, by which the lattice unit and physical unit can be converted optionally. 


$$
J *=\frac{J_{\mathrm{H}_{2}} L}{c_{\mathrm{T}} D_{\mathrm{H}_{2}-\mathrm{H}_{2} \mathrm{O}}}
$$

\subsection{Model validation}

\subsubsection{LB Model for multi-component flow}

The equi-molar counter-diffusions between $\mathrm{H}_{2}$ and $\mathrm{CO}$, as well as $\mathrm{H}_{2}$ and $\mathrm{CO}_{2}$ are employed to validate the two-component LB model established in this paper. The computation domain is a representative of the SOFC anode (as shown Fig. 2, $x=0-L$, $z=0-H)$ without the porous media. The relevant parameters are: $L=0.002 \mathrm{~m}, y_{\mathrm{H} 2 \text {,bulk }}$ $=0.8, J_{\mathrm{H} 2, \mathrm{~A} / \mathrm{E}}=0.0518 \mathrm{~mol} \cdot \mathrm{m}^{-2} \cdot \mathrm{s}^{-1}, c_{\mathrm{T}}=11.91 \mathrm{~mol} \cdot \mathrm{m}^{-3}, D_{\mathrm{H} 2-\mathrm{H} 2 \mathrm{O}}=5.44 \times 10^{-4} \mathrm{~m}^{2} \cdot \mathrm{s}^{-1}$. Therefore, $J^{*}=0.016$.

As for multi-component LB model for fluids with different molecular weights given by McCracken and Abraham[22], the ratio of the molecular weights should be below 9 in order to accurately capture the physics of binary diffusion owing to the numerical diffusion. However, as shown in Fig. 4, for both $\mathrm{H}_{2}-\mathrm{CO}$ and $\mathrm{H}_{2}-\mathrm{CO}_{2}$ systems, the molar fraction of $\mathrm{H}_{2}$ predicted by the present model agrees well with each analytical solution given by Fick's law, respectively. $M_{\mathrm{CO}} / M_{\mathrm{H}^{2}}$ and $M_{\mathrm{CO}_{2}} / M_{\mathrm{H}^{2}}$ are both larger than 9 . Therefore, the present model is not only with a better stability than that developed by McCracken and Abraham[22], but also capable of simulating two-component flow with relatively lager ratio of molecular weights.

\subsubsection{LB model for multi-component flow in porous media}

The mass transport of $\mathrm{H}_{2}, \mathrm{H}_{2} \mathrm{O}$ and $\mathrm{N}_{2}$ in an anode microstructure is employed to validate the pore scale LB model for multi-component flow in porous media. The microstructure with the porosity of $50 \%$ given by Ref.[20] is adopted to replace the 
microstructure in Fig. 2. The relevant input data are: $y_{\mathrm{H} 2 \text {,bulk }}=0.47, y_{\mathrm{H} 2 \mathrm{O} \text {,bulk }}=0.03$, $y_{\mathrm{N} 2 \text {, bulk }}=0.5, D_{\mathrm{H} 2-\mathrm{H} 2 \mathrm{O}}=3.37 \times 10^{-4} \mathrm{~m}^{2} \cdot \mathrm{s}^{-1}, D_{\mathrm{H} 2-\mathrm{N} 2}=1.085 \times 10^{-4} \mathrm{~m}^{2} \cdot \mathrm{s}^{-1}, D_{\mathrm{N} 2-\mathrm{H} 2 \mathrm{O}}=$ $0.69 \times 10^{-4} \mathrm{~m}^{2} \cdot \mathrm{s}^{-1}, T=1073 \mathrm{~K}, p=1 \mathrm{~atm}, J^{*}=0,0.032,0.064,0.096,0.128$ and 0.160 .

As shown in Fig. 5, the effect of $J^{*}$ on the concentration overpotential simulated by this paper agrees well with that by Joshi et al.[20], which ensures the accuracy of the present pore scale LB model for multi-component flow in porous media. However, Joshi et al.[20] employed the multi-component LB model developed by McCracken and Abraham[22] which is restricted by the maximum ratio of molecular weights for the simulation of multi-component flow.

\subsection{Parametric analysis}

Since different anode microstructures may have same structure statistical parameters, such as porosity, average pore size, and so on. Due to the advantage of present model, the common structure statistical parameters are not adopted in this pore scale investigation, but the effects of anode microstructures, dimensionless reaction flux and fuel composition on the mass transport within porous anode are taken into consideration.

\subsubsection{Standard case}

The input parameters of standard case are depicted in Table 1.

\subsubsection{Effect of anode microstructure}

The effect of anode microstructure is discussed from two aspects: comparing the results predicted by REV and pore scale model respectively to study the effect of the heterogeneity of the anode microstructure, and employing the pore scale LB model to 
study the mass transport in two different microstructures with the same porosity. One of the microstructures is reconstituted by the statistical method in our group represented as microstructure A, and the other is from Ref.[20] represented as microstructure B.

Fig. 6(a) (c) are the molar fraction distributions of $\mathrm{H}_{2}$ predicted respectively by the REV scale LB model developed in our previous study[6] and present pore scale LB model under different microstructures. Fig. 7 describes the mean, maximum and minimum of the molar fraction of $\mathrm{H}_{2}$ along the primary mass transport direction.

In REV scale LB model, the heterogeneous structure is converted to homogeneous structure by volume-average method, and the flow in porous media is considered as a special flow by taking into account relevant structure statistical parameters. Therefore, the molar fraction distribution of $\mathrm{H}_{2}$ has similar tendency with that of two-component equi-molar counter-diffusion, thus it decreases linearly along the primary mass transport direction (Fig. 7), and keeps constant in arbitrary cross-section perpendicular to axis $X$ (Fig. 6(a)). In pore scale LB model, the heterogeneity of porous media is considered, and the effect of the solid phase on the fluid is employed by the irregular inner boundary of the microstructure. Therefore, the molar fraction distribution of $\mathrm{H}_{2}$ varies nonlinearly along the primary mass transport direction (Fig. 7), and is no longer a constant in the cross-section perpendicular to axis $\mathrm{X}$.

For the two different microstructures with the same porosity, the mass transport within them is different under the same boundary condition. As shown in Fig. 6(b) 
and Fig. 6(c), compared with microstructure B, the size and arrangement of solid obstacles in microstructure A are much uniform, and the transport resistance is much smaller, therefore, the molar fraction of $\mathrm{H}_{2}$ is relatively bigger in the same cross-section. In the microstructure B, the size of solid obstacles changes more greatly, and the solid obstacles are much more intensive in area $\mathrm{M}\left(0.4<\mathrm{X}^{*}<0.8\right)$ in contrast to the other areas, which causes much larger transport resistance. It is clear from Fig. 7 that, in microstructure $\mathrm{B}$, the mean, maximum and minimum of molar fraction of $\mathrm{H}_{2}$ are increasingly disparate with that in microstructure $\mathrm{A}$ after $\mathrm{X}^{*}>0.4$, and the difference between the maximum and minimum is much more bigger in area $\mathrm{M}$. Notably, the concentration overpotential simulated by REV scale LB model is $0.04 \mathrm{~V}$, by pore scale LB model for microstructure $\mathrm{A}$ is $0.043 \mathrm{~V}$, and by pore scale LB model for microstructure $\mathrm{B}$ is $0.053 \mathrm{~V}$, which indicates that the more heterogeneous the microstructure is, the worse the accuracy of the REV scale model is.

Fig. 6 and Fig. 7 indicate that the heterogeneity of the anode microstructure will cause the nonlinear change of mass transport. Although with the same porosity under the same boundary condition, different mass transport will appear in different microstructures, which will affect the reaction characteristic significantly. Therefore, the anode microstructure is one of the key factors to influence the mass transport. The numerical methods, which ignore the heterogeneity, cannot describe the effect of the microstructure pattern on mass transport within the anode. The pore scale LB model established in this paper is capable of describing the mass transport in porous media precisely without the dependence of any structure statistical parameters, and 
investigating the quantitative relationship between the porous microstructure and anode performance.

\subsubsection{Effect of dimensionless reaction flux}

$J^{*}$ is employed as an influencing parameter because it has relation with some geometrical and operating parameters, such as average current density, anode thickness, pressure and temperature. Since the mass transport within microstructure A does not behave well, the size of the solid obstacles is scaled down to a microstructure with the porosity of $43 \%$, and the following parameter analyses are all based on this microstructure.

Fig. 8 describes molar fraction distributions of $\mathrm{H}_{2}$ when $J^{*}=0.006,0.018$ and 0.030 . When $L$ is a constant, a larger $J^{*}$ means a larger average current density at TPB, namely much more $\mathrm{H}_{2}$ has been consumed by the electrochemical reaction at outlet. Therefore, with increasing $J^{*}$, the molar fraction of $\mathrm{H}_{2}$ decreases more sharply along the primary mass transport direction (Fig. 8), and so does the mean value of molar fraction of $\mathrm{H}_{2}$ (Fig. 9). It is also clear in Fig. 9 that the trends of the difference between the maximum and minimum of $\mathrm{H}_{2}$ along the primary mass transport direction are similar when $J^{*}=0.006,0.018$ and 0.030 , because all the three cases are based on the same microstructure. However, the difference between the maximum and minimum of $\mathrm{H}_{2}$ increases as $J^{*}$ increases, which indicates that a larger average current density results in a less uniform concentration field. Then, more cases under different $J^{*}$ are tested as shown in Fig. 10. With increasing $J^{*}$, the average molar fraction of $\mathrm{H}_{2}$ at TPB decreases, and the concentration overpotential presents an increasing tendency 
according to Eq. (6).

\subsubsection{Effect of fuel composition}

Fig. 11 Fig. 13 depict the effect of fuel composition (molar fraction of $\mathrm{H}_{2}$ at inlet) on the mass transport in porous anode when $J^{*}=0.0001$. It is clear from Fig. 11 that the changing range of molar fraction of $\mathrm{H}_{2}$ is similar in each varying region when $y_{\mathrm{H} 2 \text {,bulk }}=0.27,0.57$ and 0.87 , separately, which results in a similar changing tendency of the mean value, maximum, minimum and difference between the maximum and minimum of the molar fraction of $\mathrm{H}_{2}$ along the primary mass transport direction (Fig. 12). It is because that all those three cases are based on the same microstructure and $J^{*}$. Then, more cases under different $y_{\mathrm{H} 2 \text {,bulk }}$ are tested as shown in Fig. 13. With increasing the molar fraction of $\mathrm{H}_{2}$ at inlet, the average molar fraction of $\mathrm{H}_{2}$ at TPB increases since the molar flux of $\mathrm{H}_{2}$ keeps constant, and according to Eq. (6), the concentration overpotential firstly decreases, then increases. It agrees well with the conclusion given by Ref.[6] using the REV scale LB model, which assures the reliability of the present pore scale LB model for multi-component flow in porous anode over again.

\section{Conclusion}

In this paper, a two-fluid finite-difference LB model for multi-component flow is proposed and proved to be capable of simulating two-component flow with relatively lager ratio of molecular weights so as to meet the fuel flexibility of SOFC, since the employed finite-difference scheme for streaming process could mitigate the numerical 
diffusion effectively in contrast to the existing interpolation scheme. Furthermore, a pore scale LB model for multi-component flow in porous media is established based on the newly built two-fluid finite-difference LB model to investigate the gaseous mixture flow in the porous anode. The characteristics of gas transport are quite different in different microstructures with the same structure statistical parameters, which indicates that the heterogeneity of electrode microstructures significantly affect the transport processes in the anode. The numerical methods assuming that the porous media is homogeneous are not competent to predict the different cell performance resulted from the heterogeneity of microstructures. Moreover, the pore scale model presented in this study is adopted to investigate the effects of dimensionless reaction flux and fuel composition so as to reveal the influence of anode microstructure on the concentration overpotential characteristics of SOFC. The method and results presented in this study can be helpful for investigation of multi-component mass transport in porous electrodes and for optimization of electrochemical performance of SOFC.

\section{Nomenclature}

a Acceleration, $\mathrm{m} \cdot \mathrm{s}^{-2}$

c Total molar concentration, $\mathrm{mol} \cdot \mathrm{m}^{-3}$

$D_{i j} \quad$ Diffusion coefficient between species $i$ and species $j, \mathrm{~m}^{2} \cdot \mathrm{s}^{-1}$

$f \quad$ Density distribution function

F Faraday constant, $96485 \mathrm{C} \cdot \mathrm{mol}^{-1}$ 
$i \quad$ Current density, $\mathrm{A} \cdot \mathrm{m}^{-2}$

$J \quad$ Mass flux, $\mathrm{kg} \cdot \mathrm{m}^{-3} \cdot \mathrm{s}^{-1}$

$J^{\sigma \sigma} \quad$ Self-collision term

$J^{\sigma s} \quad$ Cross-collision term

L Thickness, m

$M \quad$ Molecular weight, $\mathrm{kg} \cdot \mathrm{mol}^{-1}$

$p \quad$ Pressure, $\mathrm{Pa}$

$R \quad$ Gas constant, $\mathrm{J} \cdot \mathrm{mol}^{-1} \cdot \mathrm{K}^{-1}$

$t \quad$ Time, $\mathrm{s}$

$T \quad$ Temperature, $\mathrm{K}$

$\boldsymbol{u} \quad$ Velocity, $\mathrm{m} \cdot \mathrm{s}^{-1}$

$\eta_{c o n} \quad$ Concentration overpotential, $\mathrm{V}$

$y_{i} \quad$ Molar fraction of species $i$

Greek symbols

$\rho \quad$ Density, $\mathrm{kg} \cdot \mathrm{m}^{-3}$

Subscripts

a Anode

$\mathrm{A} / \mathrm{E} \quad$ Interface between anode and electrolyte

Bulk Gas channel

e Electrolyte

Superscript

$\sigma \quad$ Species $\sigma$ 
* Non-dimensional

\section{Acknowledgments}

This work is supported by National Natural Science Foundation of China (50976092), Scientific and Technological Project for Industry in Shaanxi Province, China (2015GY096), National Science Foundation for Post-doctoral Scientists of China (2015M582651) and Scientific Research Foundation for Lecturers of Xi'an Jiaotong University.

\section{References}

[1]F. Yang, J. Gu, L. Ye, Z. Zhang, G. Rao, Y. Liang, K. Wen, J. Zhao, J.B. Goodenough, W. He, Justifying the significance of Knudsen diffusion in solid oxide fuel cells, Energy, 95(2016) 242-246.

[2]D. Papurello, A. Lanzini, D. Drago, P. Leone, M. Santarelli, Limiting factors for planar solid oxide fuel cells under different trace compound concentrations, Energy, 95(2016) 67-78.

[3]Q. Chen, Q. Wang, J. Zhang, J. Yuan, Effect of bi-layer interconnector design on mass transfer performance in porous anode of solid oxide fuel cells, International Journal of Heat and Mass Transfer, 54(2011) 1994-2003.

[4]Q. Cai, C.S. Adjiman, N.P. Brandon, Investigation of the active thickness of solid oxide fuel cell electrodes using a 3D microstructure model, Electrochimica Acta, 56(2011) 10809-10819. 
[5]S. Tonekabonimoghadam, R.K. Akikur, M.A. Hussain, S. Hajimolana, R. Saidur, H.W. Ping, M.H. Chakrabarti, N.P. Brandon, P.V. Aravind, J.N.S. Nayagar, M.A. Hashim, Mathematical modelling and experimental validation of an anode-supported tubular solid oxide fuel cell for heat and power generation, Energy, 90(2015) $1759-1768$.

[6]H. Xu, Z. Dang, B. Bai, Numerical Simulation of Multispecies Mass Transfer in a SOFC Electrodes Layer Using Lattice Boltzmann Method, Journal of Fuel Cell Science and Technology, 9(2012) 61004.

[7]I. EG G Technical Services, Fuel Cell Handbook, 7 ed., Morgantown, West Virginia, 2004.

[8]A. Baldinelli, L. Barelli, G. Bidini, Performance characterization and modelling of syngas-fed SOFCs (solid oxide fuel cells) varying fuel composition, Energy, 90(2015) 2070-2084.

[9]H. Iwai, Y. Yamamoto, M. Saito, H. Yoshida, Numerical simulation of intermediate-temperature direct-internal-reforming planar solid oxide fuel cell, Energy, 36(2011) 2225-2234.

[10]J.R. Wilson, W. Kobsiriphat, R. Mendoza, H. Chen, J.M. Hiller, D.J. Miller, K. Thornton, P.W. Voorhees, S.B. Adler, S.A. Barnett, Three-dimensional reconstruction of a solid-oxide fuel-cell anode, Nature Materials, 5(2006) 541-544.

[11]S.H. Chan, Z.T. Xia, Anode Micro Model of Solid Oxide Fuel Cell, Journal of the Electrochemical Society, 148(2001) A388-A394.

[12]M. Kishimoto, H. Iwai, M. Saito, H. Yoshida, Three-Dimensional Simulation of 
SOFC Anode Polarization Characteristics Based on Sub-Grid Scale Modeling of Microstructure, Journal of the Electrochemical Society, 159(2012) B315-B323.

[13]H. Yakabe, M. Hishinuma, M. Uratani, Y. Matsuzaki, I. Yasuda, Evaluation and modeling of performance of anode-supported solid oxide fuel cell, Journal of Power Sources, 86(2000) 423-431.

[14]R. Suwanwarangkul, E. Croiset, M.W. Fowler, P.L. Douglas, E. Entchev, M.A. Douglas, Performance comparison of Fick's, dusty-gas and Stefan - Maxwell models to predict the concentration overpotential of a SOFC anode, Journal of Power Sources, 122(2003) 9-18.

[15]M. Ni, M.K.H. Leung, D.Y.C. Leung, Parametric study of solid oxide fuel cell performance, Energy Conversion and Management, 48(2007) 1525-1535.

[16]H.R. Amedi, B. Bazooyar, M.R. Pishvaie, Control of anode supported SOFCs (solid oxide fuel cells): Part I. mathematical modeling and state estimation within one cell, Energy, 90(2015) 605-621.

[17]Y. Suzue, N. Shikazono, N. Kasagi, Micro modeling of solid oxide fuel cell anode based on stochastic reconstruction, Journal of Power Sources, 184(2008) 52-59. [18]D. Kanno, N. Shikazono, N. Takagi, K. Matsuzaki, N. Kasagi, Evaluation of SOFC anode polarization simulation using three-dimensional microstructures reconstructed by FIB tomography, Electrochimica Acta, 56(2011) 4015-4021.

[19]P. Asinari, M.C. Quaglia, M.R. von Spakovsky, B.V. Kasula, Direct numerical calculation of the kinematic tortuosity of reactive mixture flow in the anode layer of solid oxide fuel cells by the lattice Boltzmann method, Journal of Power Sources, 
170(2007) 359-375.

[20]A.S. Joshi, K.N. Grew, A.A. Peracchio, W. Chiu, Lattice Boltzmann modeling of 2D gas transport in a solid oxide fuel cell anode, Journal of Power Sources, 164(2007) $631-638$

[21]K.N. Grew, A.S. Joshi, A.A. Peracchio, W. Chiu, Pore-scale investigation of mass transport and electrochemistry in a solid oxide fuel cell anode, Journal of Power Sources, 195(2010) 2331-2345.

[22]M.E. McCracken, J. Abraham, Lattice Boltzmann methods for binary mixtures with different molecular weights, Physical Review E, 71(2005) 46704.

[23]H. Xu, Z. Dang, B. Bai, Electrochemical performance study of solid oxide fuel cell using lattice Boltzmann method, Energy, 67(2014) 575-583.

[24]W. Chiu, A.S. Joshi, K.N. Grew, Lattice Boltzmann model for multi-component mass transfer in a solid oxide fuel cell anode with heterogeneous internal reformation and electrochemistry, European Physical Journal-Special Topics, 171(2009) 159-165. [25]S.H. Chan, K.A. Khor, Z.T. Xia, A complete polarization model of a solid oxide fuel cell and its sensitivity to the change of cell component thickness, Journal of Power Sources, 93(2001) 130-140.

[26]Y. Shi, N. Cai, C. Li, C. Bao, E. Croiset, J. Qian, Q. Hu, S. Wang, Modeling of an anode-supported Ni-YSZ|Ni-ScSZ|ScSZ|LSM-ScSZ multiple layers SOFC cell: Part I. Experiments, model development and validation, Journal of Power Sources, 172(2007) 235-245.

[27]L. Luo, S.S. Girimaji, Theory of the lattice Boltzmann method: Two-fluid model 
for binary mixtures, Physical Review E, 67(2003) 36302.

[28]L. Luo, S.S. Girimaji, Lattice Boltzmann model for binary mixtures, Physical Review E, 66(2002) 35301.

[29]T.S. Zhao, Z. Guo, Explicit finite-difference lattice Boltzmann method for curvilinear coordinates, Physical Review E, 67(2003) 66709.

[30]F. Nannelli, S. Succi, The lattice Boltzmann equation on irregular lattices, Journal of Statistical Physics, 68(1992) 401-407.

[31]R.M. MacMeccan, J.R. Clausen, G.P. Neitzel, C.K. Aidun, Simulating deformable particle suspensions using a coupled lattice-Boltzmann and finite-element method, Journal of Fluid Mechanics, 618(2009) 13-39.

[32]A.S. Joshi, A.A. Peracchio, K.N. Grew, W.K.S. Chiu, Lattice Boltzmann method for continuum, multi-component mass diffusion in complex 2D geometries, Journal of Physics D: Applied Physics, 40(2007) 2961-2971.

[33]S. S, The Lattice Boltzmann equation for fluid dynamics and beyond, Oxford: Clarendon Press, 2001. 


\section{List of Figurers}

1. Fig. 1 (a) Schematic of the multi-component mass transport and electrochemical reaction in an SOFC anode (b) A SEM image of the anode microstructure (c) A binary image of the anode microstructure after binarization processing.

2. Fig. 2 Physical model and boundary conditions of mass transport in anode.

3. Fig. 3 Schematic of inner boundary treatment.

4. Fig. 4 Molar fraction of $\mathrm{H}_{2}$ under different fuel composition.

5. Fig. 5 Effect of $J^{*}$ on concentration overpotential.

6. Fig. 6 Molar fraction distributions of $\mathrm{H}_{2}$ predicted by REV scale LB model and pore scale LB model under different microstructures $(\varepsilon=35 \%)$.

7. Fig. 7 Molar fraction of $\mathrm{H}_{2}$ along the primary mass transport direction predicted by REV and pore scale LB model under different microstructures $(\varepsilon=35 \%)$.

8. Fig. 8 Effect of $J^{*}$ on molar fraction distributions of $\mathrm{H}_{2}$ within the anode.

9. Fig. 9 Effect of $J^{*}$ on molar fraction of $\mathrm{H}_{2}$ along the primary mass transport direction.

10. Fig. 10 Effect of $J^{*}$ on average molar fraction of $\mathrm{H}_{2}$ at TPB and concentration overpotential.

11. Fig. 11 Effect of molar fraction of $\mathrm{H}_{2}$ at inlet on molar fraction distributions of $\mathrm{H}_{2}$ within the anode.

12. Fig. 12 Effect of molar fraction of $\mathrm{H}_{2}$ at inlet on molar fraction of $\mathrm{H}_{2}$ along the primary mass transport direction.

13. Fig. 13 Effect of molar fraction of $\mathrm{H}_{2}$ at inlet on average molar fraction of $\mathrm{H}_{2}$ at 
TPB and concentration overpotential. 


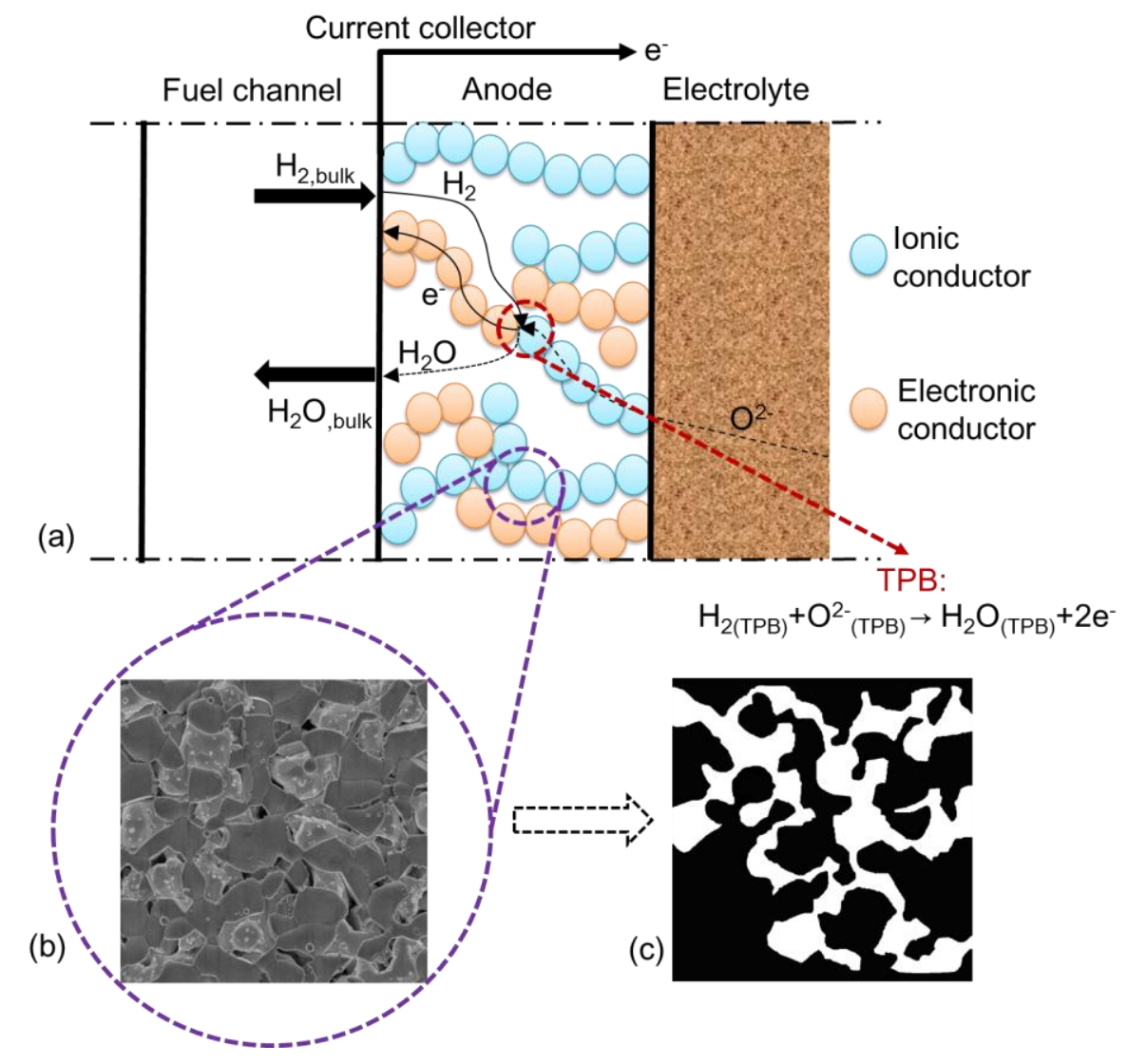

Fig. 1 (a) Schematic of the multi-component mass transport and electrochemical reaction in an SOFC anode (b) A SEM image of the anode microstructure (c) A binary image of the anode microstructure after binarization processing. 


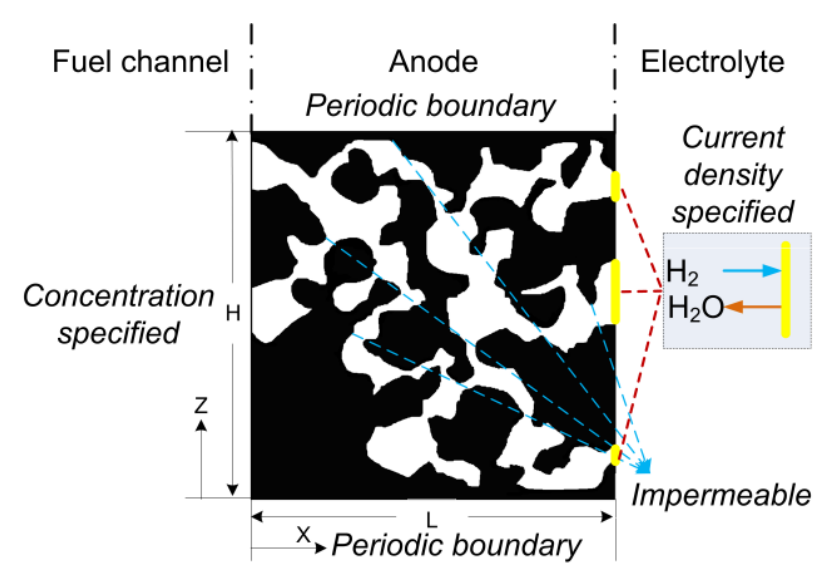

Fig. 2 Physical model and boundary conditions of mass transport in anode. 


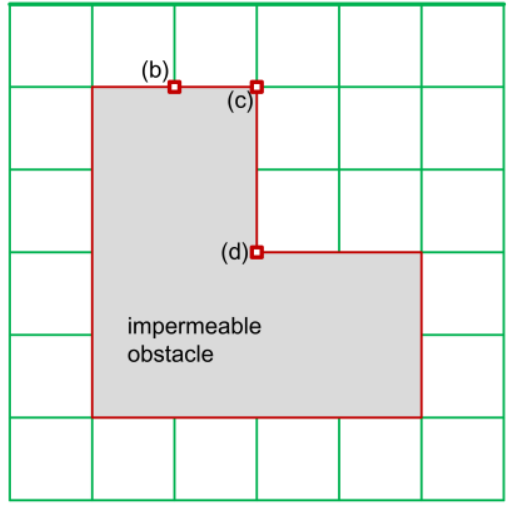

(a) "Grids" area represents the pore phase; "Gray" area represents the solid phase; "Inner boundary" line represents the interface.

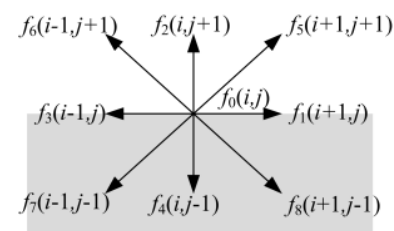

(b) Flat boundary

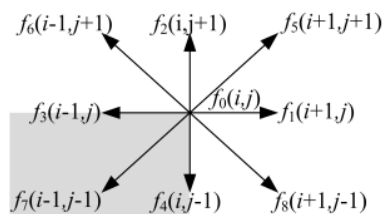

(c) Convex boundary

$f_{7}(i-1, j-1) \quad f_{4}(i, j-1) \quad f_{8}(i+1, j-1)$

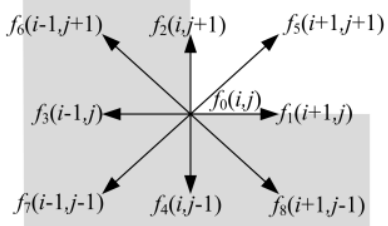

(d) Concave boundary

Fig. 3 Schematic of inner boundary treatment. 


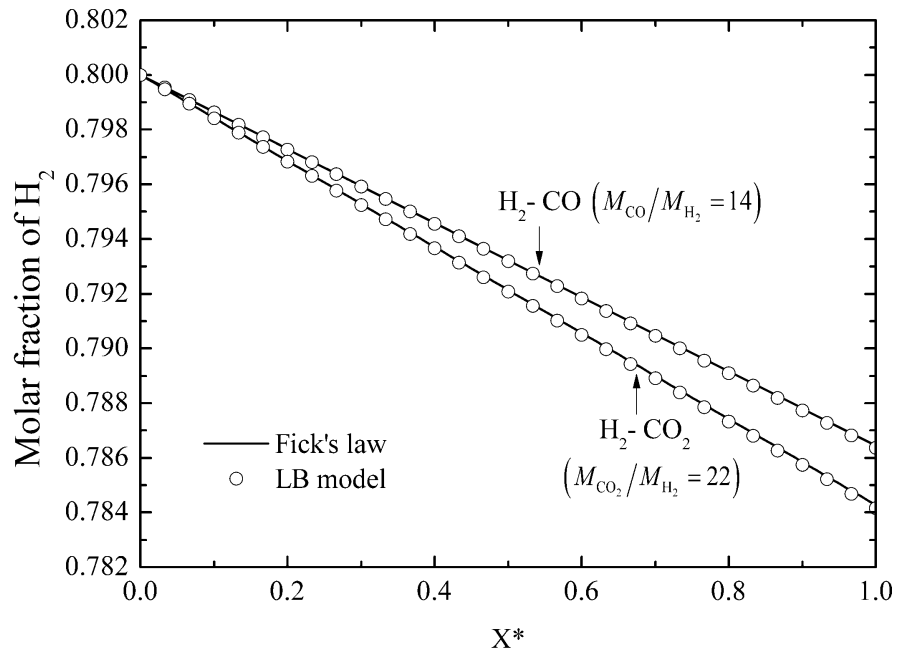

Fig. 4 Molar fraction of $\mathrm{H}_{2}$ under different fuel composition. 


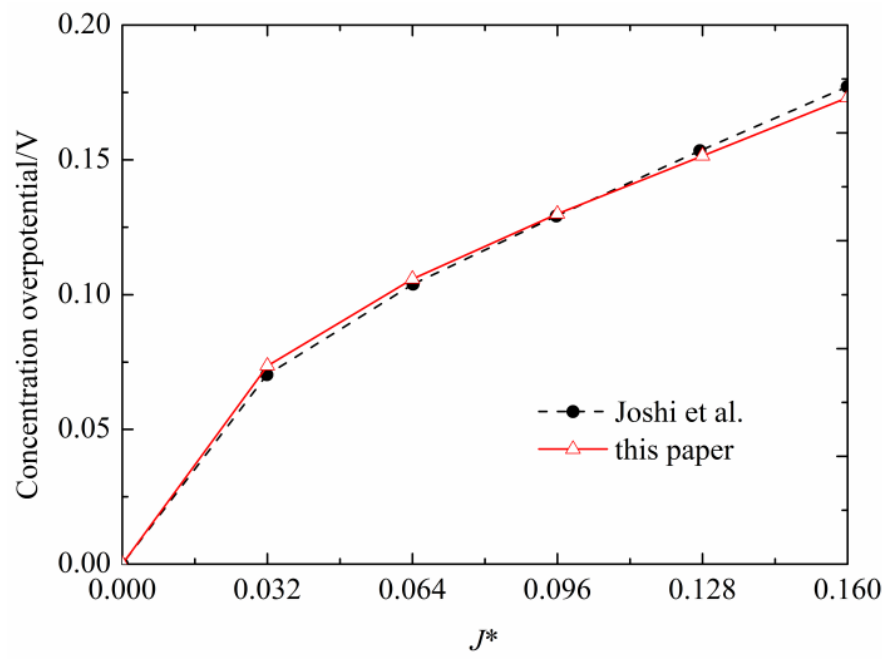

Fig. 5 Effect of $J^{*}$ on concentration overpotential. 


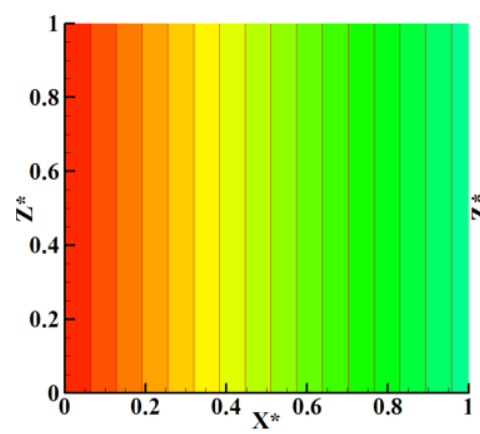

(a) REV scale

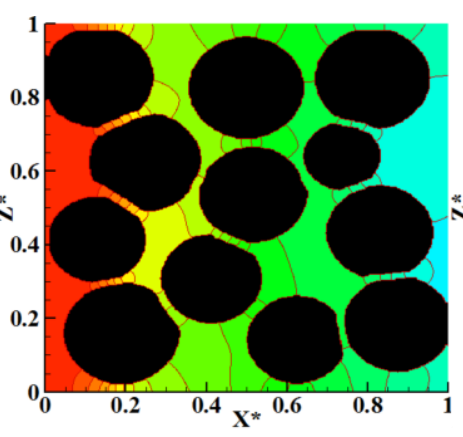

(b) Pore scale(microstructure A)

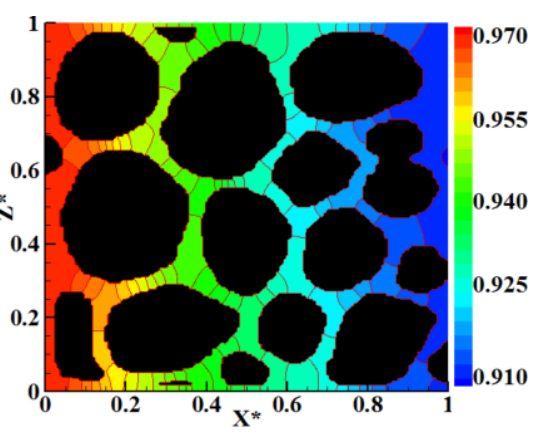

(c) Pore scale(microstructure B)

Fig. 6 Molar fraction distributions of $\mathrm{H}_{2}$ predicted by REV scale LB model and pore scale LB model under different microstructures $(\varepsilon=35 \%)$. 


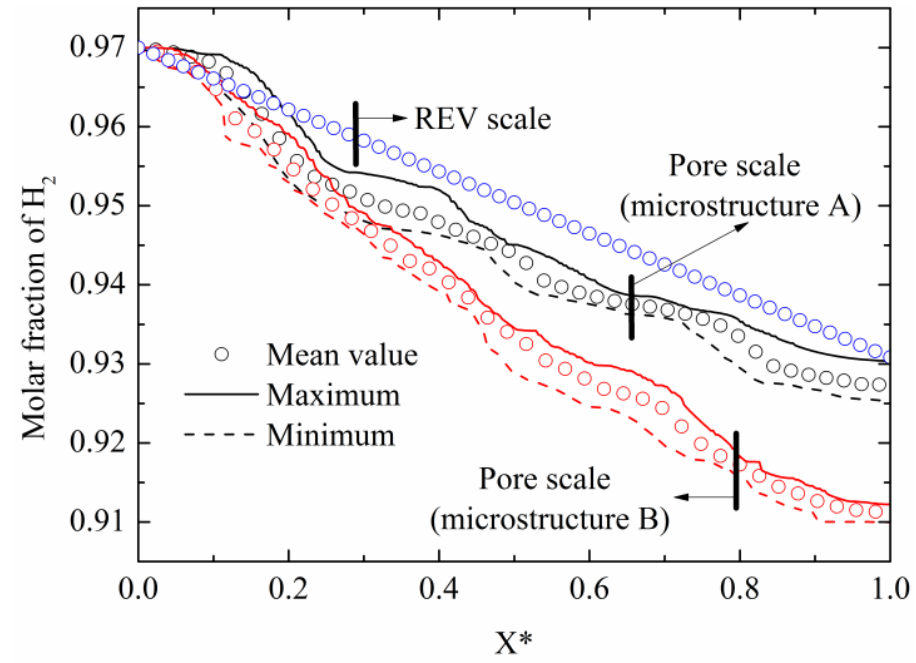

Fig. 7 Molar fraction of $\mathrm{H}_{2}$ along the primary mass transport direction predicted by

REV and pore scale LB model under different microstructures $(\varepsilon=35 \%)$. 


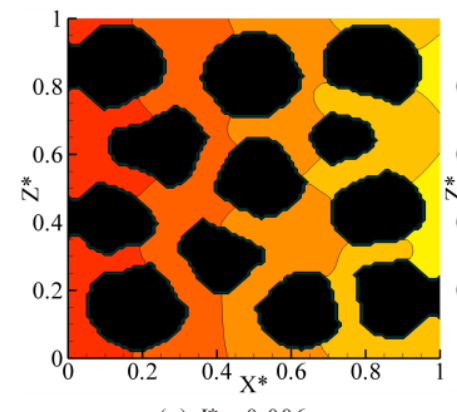

(a) $J^{*}=0.006$

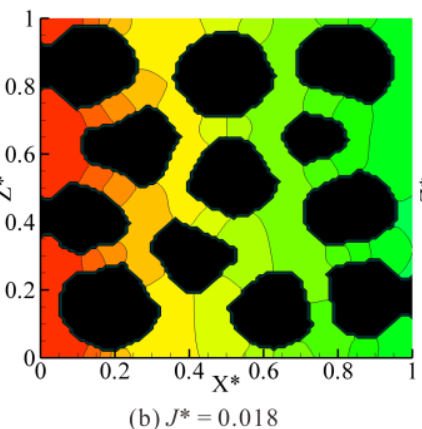

(b) $J^{*}=0.018$

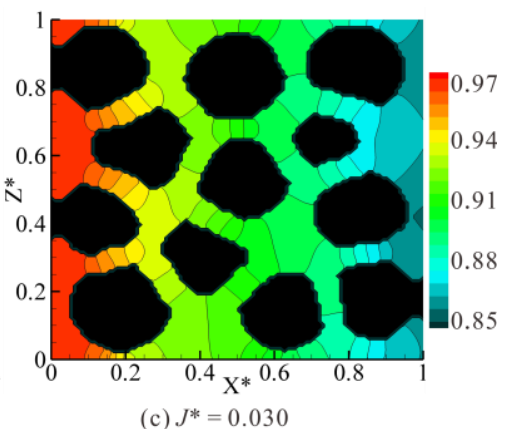

(c) $J^{*}=0.030$

Fig. 8 Effect of $J^{*}$ on molar fraction distributions of $\mathrm{H}_{2}$ within the anode. 


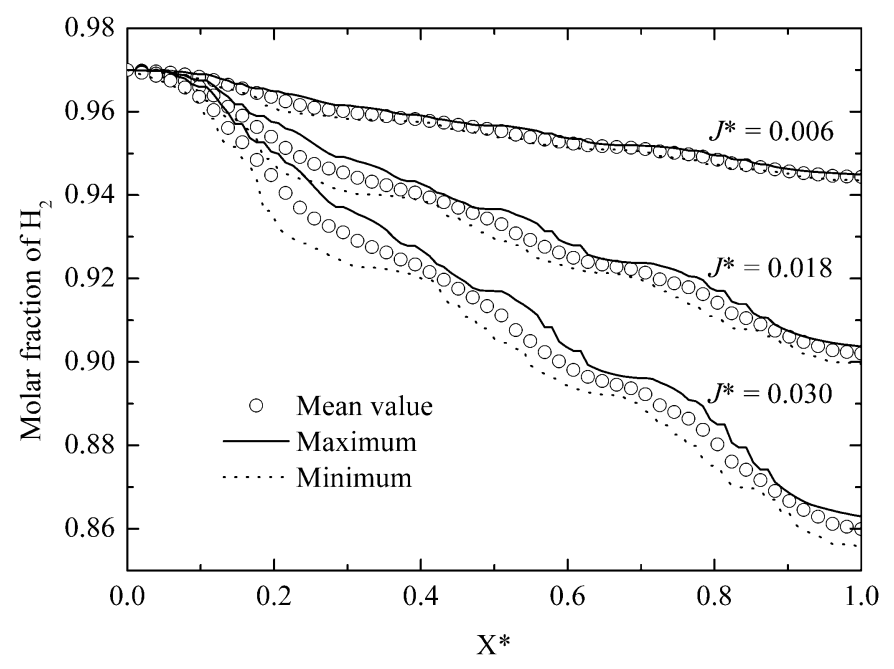

Fig. 9 Effect of $J^{*}$ on molar fraction of $\mathrm{H}_{2}$ along the primary mass transport direction. 


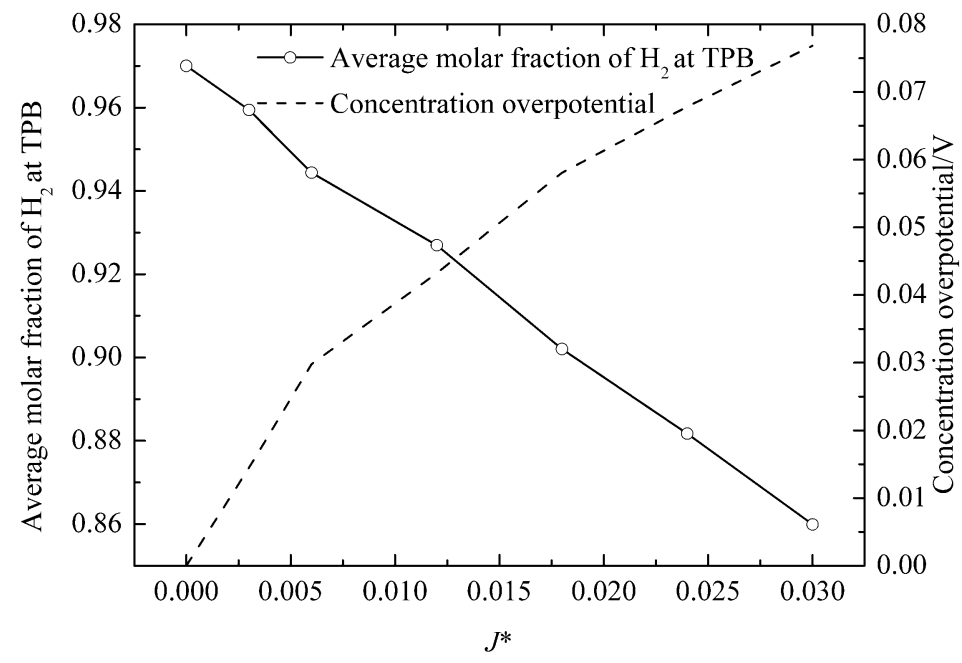

Fig. 10 Effect of $J^{*}$ on average molar fraction of $\mathrm{H}_{2}$ at TPB and concentration overpotential. 


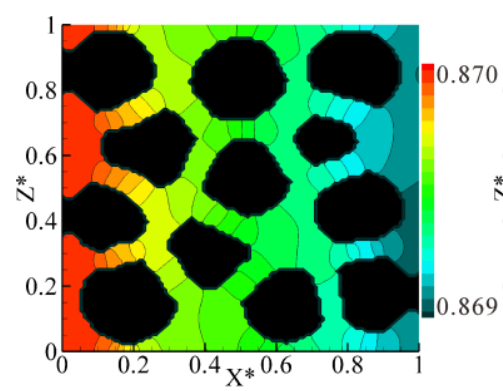

(a) $y_{\text {H,bulk }}=0.87$

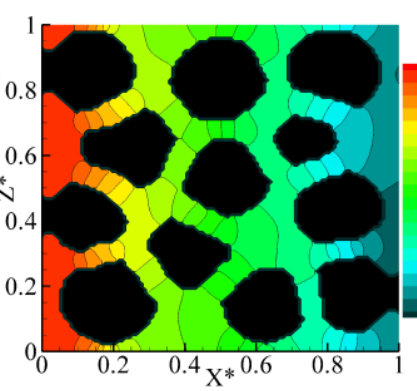

(b) $y_{\text {H,bulk }}=0.57$

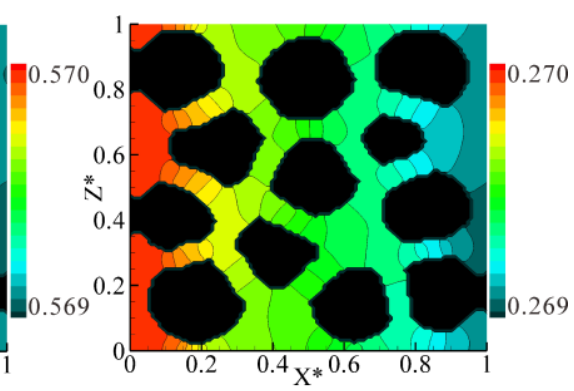

(c) $y_{\text {H,bulk }}=0.27$

Fig. 11 Effect of molar fraction of $\mathrm{H}_{2}$ at inlet on molar fraction distributions of $\mathrm{H}_{2}$

within the anode. 


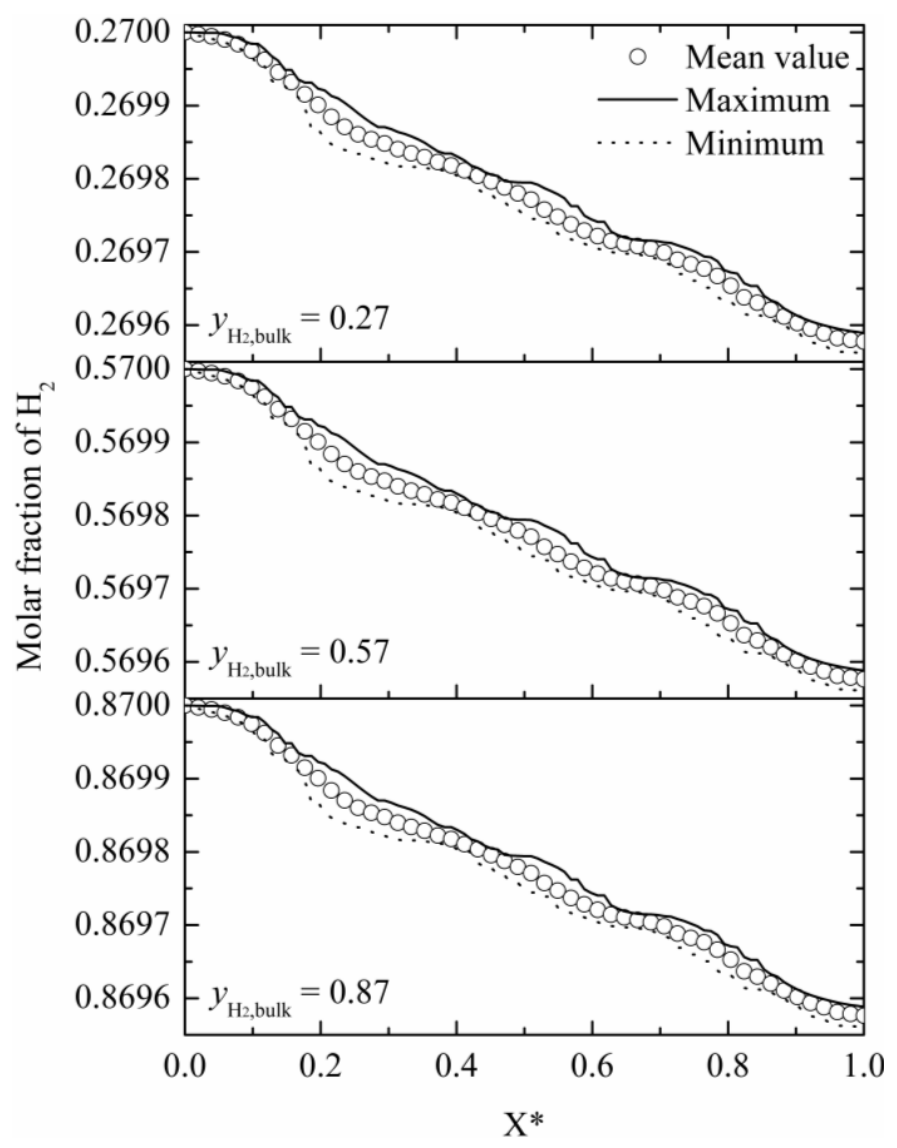

Fig. 12 Effect of molar fraction of $\mathrm{H}_{2}$ at inlet on molar fraction of $\mathrm{H}_{2}$ along the primary mass transport direction. 


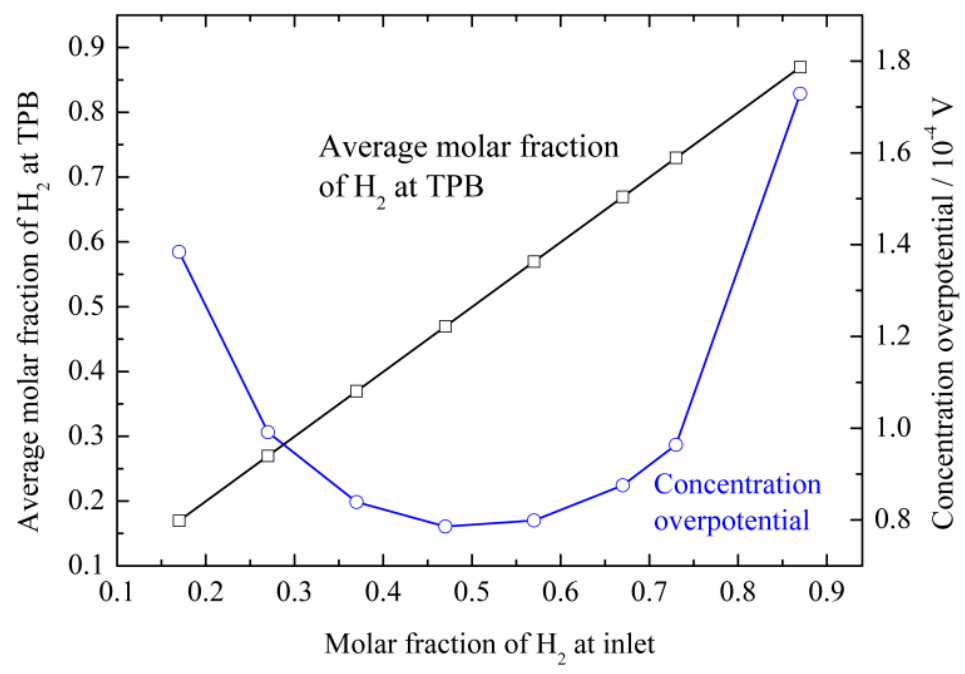

Fig. 13 Effect of molar fraction of $\mathrm{H}_{2}$ at inlet on average molar fraction of $\mathrm{H}_{2}$ at TPB and concentration overpotential. 


\section{List of Tables}

1. Table 1 Input parameters of standard case. 
Table 1 Input parameters of standard case.

\begin{tabular}{ll}
\hline Parameter & Value \\
\hline Operating temperature / K & 1073 \\
Operating pressure / Pa & 101325 \\
Fuel composition & $97 \% \mathrm{H}_{2}, 0.3 \% \mathrm{H}_{2} \mathrm{O}$ \\
Average current density / $\mathrm{A} \mathrm{m}^{-2}$ & 3000 \\
Anode thickness / $\mathrm{m}$ & $750 \times 10^{-6}$ \\
\hline
\end{tabular}

